\title{
Research on the Recognition of Paper Currency Number with Low Resolution
}

\author{
Xiwen Liu ${ }^{1,}$, Chaoying Liu ${ }^{1, b}$ \\ ${ }^{1}$ Department of Electronic Information \& Mechanical Engineering, Zhaoqing University, \\ Zhaoqing, Guangdong, 526061, China \\ aemail: liuxiwen1111@163.com, bemail:chaoying1221@126.com
}

Keywords: Serial number; Character extraction; Multi-level 2DPCA; Pattern recognition

\begin{abstract}
According to the characteristics that the paper currency image has low resolution and it is vulnerable to noise affect, the methods of paper serial number region extraction and segmentation are discussed, and multi-level 2DPCA algorithm is used for serial number recognition. Experiment results show that the methods proposed in this paper have the advantages of fast speed, high recognition accuracy and good anti-interference ability.
\end{abstract}

\section{Introduction}

The serial numbers of paper currency identify each note. It is significant to recognize these numbers for preventing forged bills. As Contact Image Sensor (CIS) has no optical lens, it has small size and simple structure. At present, CIS is often applied for getting paper currency image [1,2]. In order to increase image scanning speed, the bill's CIS image always has low resolution.

As for paper currency serial number recognition, the technologies such as template matching and artificial neural network are usually used $[3,4]$. The template matching method is not suitable for characters with low resolution or polluted characters. Artificial neural network needs plenty of training samples, and it has low recognition accuracy to untrained images. Therefore, multi-level 2DPCA algorithm is proposed for paper currency serial number recognition in this paper, this algorithm has the advantages of fast speed, high recognition accuracy and good anti-interference ability.

\section{Extraction of paper currency serial number}

As shown in Fig.1, the paper currency image is acquired by CIS whose resolution is 100DPI. As the resolution of CIS is low and the paper currency is polluted, it can be seen that the serial number is slightly fuzzy; and no obvious noises exist around the serial number.

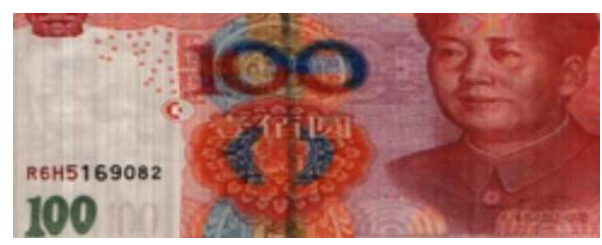

Fig.1 Paper currency CIS image

Sobel operator is used for edge extraction first, and then closing operation of mathematical morphology is applied for serial number region enhancement. The resulted image is shown in Fig.2(b), the serial number region can be easily found in this image.

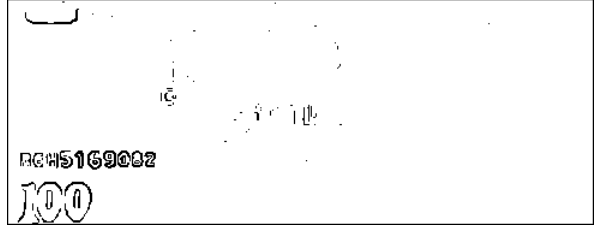

(a) Sobel operator edge extraction

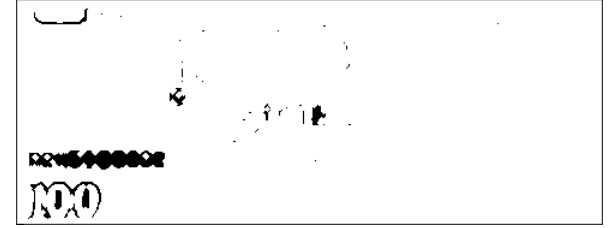

(b) closing operation of mathematical morphology

Fig.2 Serial number region extraction 
As shown in Fig.3, convert Fig.1 into binary image; and then scan the serial number region, it is not difficult to extract the characters. If the quantity of characters is not equal to ten, the extraction is failed.

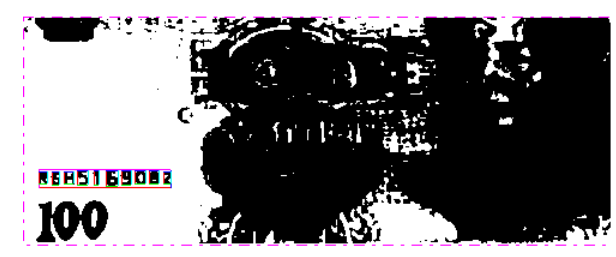

Fig.3 Extraction of serial number

\section{PCA and 2DPCA recognition}

\section{PCA recognition.}

Principal Components Analysis (PCA) is one of the most popular linear mapping methods in pattern recognition. The principal components are the linear combination of the original data, and they are linearly independent. The principal component's eigenvalues represent variances. The bigger the variance is, the more important the principal component is. If the principal component's variance is very little, it contains noisy, and it should be discarded in analysis; therefore, the number of principal component for analyzing is decreased, and the data dimension is reduced. Usually, the principal components with big eigenvalue are selected for analysis.

The steps of PCA analysis are as follows.

Assume the sample matrix is $X=\left(\begin{array}{cccc}x_{11} & x_{12} & \cdots & x_{1 p} \\ x_{21} & x_{22} & \cdots & x_{2 p} \\ \vdots & \vdots & \vdots & \vdots \\ x_{n 1} & x_{n 2} & \cdots & x_{n p}\end{array}\right)$

Step 1: Get the standard matrix $X^{*}$ by processing original data.

$$
x_{i j}^{*}=\frac{x_{i j}-\bar{x}_{j}}{\sqrt{\operatorname{var}\left(x_{j}\right)}} \quad(i=1,2, \cdots, n ; j=1,2, \cdots, p)
$$

In the equation above,

$$
\begin{aligned}
& \bar{x}_{j}=\frac{1}{n} \sum_{i=1}^{n} x_{i j} \\
& \operatorname{var}\left(x_{j}\right)=\frac{1}{n-1} \sum_{i=1}^{n}\left(x_{i j}-\bar{x}_{j}\right)^{2} \quad(j=1,2, \cdots, p)
\end{aligned}
$$

Step 2 :Calculate sample's correlation coefficient matrix.

$$
R=\left[\begin{array}{cccc}
r_{11} & r_{12} & \cdots & r_{1 p} \\
r_{21} & r_{22} & \cdots & r_{2 p} \\
\vdots & \vdots & \cdots & \vdots \\
r_{p 1} & r_{p 2} & \cdots & r_{p p}
\end{array}\right]
$$

The correlation coefficient is calculated as

$$
r_{i j}=\frac{1}{n-1} \sum_{t=1}^{n} x_{t i}^{*} x_{t j}^{*} \quad(i, j=1,2, \cdots, p)
$$

Step 3:Calculate the eigenvalues $\left(\lambda_{1}, \lambda_{2} \cdots \lambda_{p}\right)$ and responding feature vectors of correlation 
coefficient matrix $R$.

Step 4:Select important principal components and give their equations.

The main shortcoming of PCA algorithm is that it has a large scale of calculation. And in recent years, there are many improved algorithms proposed to overcome the shortcoming. Two-Dimensional Principal Component Analysis (2DPCA) is one of the improved algorithms.

\section{DPCA recognition.}

The feature extraction of 2DPCA is directly based on image matrix; therefore, its calculation is small, and its speed is obvious faster than that of PCA [6,7]. Assume the dimension of image $X$ is $m \times n$, projection space $\mathrm{U} \in R^{n \times p}, n \geq p$. When the image matrix $X$ is projected onto $U$, a new matrix $Y=X U$ whose dimension is $m \times p$ should be produced. In 2DPCA, the total dispersion of projection matrix is used as criterion function $J(U)$ to measure the quality of the projection space.

$$
J(U)=\operatorname{tr}\left(S_{U}\right)
$$

In the equation above, $S_{U}$ is covariance matrix of projection matrix $Y, \operatorname{tr}\left(S_{U}\right)$ is the trace of $S_{U} \cdot$

$$
S_{U}=U^{T} G U
$$

$G$ is the covariance matrix of image $X$, and $G$ is defined as follows:

$$
G=E\left\{[x-E(x)]^{T}[x-E(x)]\right\}
$$

$\mathrm{G}$ is a $n \times n$ positive matrix. Assume $x_{i}(i=1,2, \ldots, M)$ is testing image, the average matrix $U$ and the covariance matrix $G$ are as follows:

$$
U=\frac{1}{M} \sum_{i=1}^{M} x_{i}, G=\frac{1}{M} \sum_{i=1}^{M}\left(x_{i}-u\right)^{T}\left(x_{i}-u\right)
$$

The subsequent calculating steps are same as PCA algorithm.

\section{Multi-level 2DPCA characters recognition.}

In the paper currency image, some characters have low resolution and some characters are similar, these conditions will lead to inaccurate recognition; in order to solve the problem, the similar characters are grouped for multi-level recognition. The grouped characters are shown in Table 1.

Table 1 Groups of similar characters

\begin{tabular}{|c|c|c|c|}
\hline Groups(First level) & \multicolumn{3}{|c|}{ Characters } \\
\hline \multirow{2}{*}{1} & \multirow{3}{*}{$0,3,6,8,9, \mathrm{~B}}$, & Sub-groups(Second level) & Characters \\
\cline { 3 - 4 } & \multirow{2}{*}{$\mathrm{C}, \mathrm{D}, \mathrm{G}, \mathrm{O}, \mathrm{Q}$} & 1 & $8, \mathrm{~B}$ \\
\cline { 3 - 4 } & & 2 & 3,9 \\
\cline { 3 - 4 } & & 3 & $6, \mathrm{C}, \mathrm{D}, \mathrm{G}$ \\
\hline 2 & & 4 & $0, \mathrm{O}, \mathrm{Q}$ \\
\hline 3 & & \multicolumn{3}{|c|}{$\mathrm{M}, \mathrm{N}, \mathrm{H}$} \\
\hline
\end{tabular}

As shown in Table 1, the similar characters are divided into three groups which are belong to first level, and group 1 is divided into four sub-groups which are belong to second level. The steps of multi-2DPCA recognition are as follows:

(1) Carry out 2DPCA recognition by using all bill character as training samples.

(2) If the recognition result is one of similar characters which are shown in first level groups, use the same group characters with the recognition result as training samples and carry out 2DPCA recognition.

(3) If the previous recognition result is one of the similar characters which are shown in secondary level groups, carry out 2DPCA recognition by using the same group characters with the recognition result as training samples. 


\section{Experiment results}

Extract characters 0-9, A-Z from paper currency image, there are 36 kinds of characters, collect five samples for every kind character. Therefore, there are totally 180 samples. The bill characters have the following features.

(1) The size of characters is not unique.

(2) The character's height is bigger than width; the height is about 1.6 times the width.

(3) Among the same character sets, some are thick, some are thin.

For the sake of above features, the character must be normalized first, the size of normalized character is $20 \times 32$, therefore, the normalized character has 640 pixels. If PCA algorithm is applied for recognition, calculation is large and long time will be cost. So, 2DPCA algorithm is applied for feature extraction, decrease the feature dimension to 100 first, and then template matching is applied for recognition.

Pixel template matching, PCA and 2DPCA are used for character recognition respectively; the testing samples include wear bills, polluted bills, new and old bills, and they are well-distributed. The recognition results are shown in Table 2.

Table 2 Results of recognition with pixel template matching,PCA and 2DPCA methods

\begin{tabular}{|c|c|c|c|c|c|c|c|}
\hline \multirow{3}{*}{ Character } & \multirow{3}{*}{ Quantity } & \multicolumn{6}{|c|}{ Errors } \\
\hline & & \multicolumn{2}{|c|}{ Template matching } & \multicolumn{2}{|c|}{ PCA } & \multicolumn{2}{|c|}{ 2DPCA } \\
\hline & & Quantity & Ratio (\%) & Quantity & Ratio (\%) & Quantity & Ratio $(\%)$ \\
\hline 0 & 102 & 45 & 44.4 & 20 & 19.6 & 8 & 7.84 \\
\hline 1 & 85 & 40 & 47.6 & 18 & 21.2 & 2 & 2.36 \\
\hline 2 & 75 & 30 & 40 & 8 & 10.67 & 1 & 1.33 \\
\hline 3 & 86 & 42 & 48.84 & 14 & 16.28 & 5 & 5.81 \\
\hline 4 & 75 & 35 & 46.67 & 4 & 5.34 & 1 & 1.33 \\
\hline 5 & 70 & 30 & 42.86 & 4 & 5.71 & 1 & 1.42 \\
\hline 6 & 80 & 38 & 47.5 & 9 & 11.25 & 4 & 5.0 \\
\hline 7 & 92 & 20 & 21.74 & 5 & 5.43 & 1 & 1.07 \\
\hline 8 & 78 & 41 & 52.56 & 25 & 32.05 & 9 & 11.54 \\
\hline 9 & 95 & 42 & 44.21 & 18 & 18.95 & 4 & 4.21 \\
\hline $\mathrm{A}$ & 52 & 16 & 30.77 & 5 & 9.62 & 1 & 1.92 \\
\hline B & 49 & 15 & 30.61 & 12 & 24.49 & 5 & 10.20 \\
\hline $\mathrm{C}$ & 38 & 10 & 26.32 & 6 & 15.79 & 4 & 10.53 \\
\hline $\mathrm{D}$ & 45 & 16 & 35.56 & 8 & 17.78 & 6 & 13.33 \\
\hline$E$ & 46 & 14 & 30.43 & 6 & 13.04 & 1 & 2.17 \\
\hline $\mathrm{F}$ & 42 & 10 & 23.80 & 2 & 4.76 & 0 & 0 \\
\hline $\mathrm{G}$ & 39 & 11 & 28.81 & 4 & 10.26 & 2 & 5.12 \\
\hline $\mathrm{H}$ & 38 & 10 & 26.3 & 3 & 7.89 & 1 & 2.63 \\
\hline I & 40 & 10 & 25.0 & 3 & 7.5 & 0 & 0 \\
\hline $\mathrm{J}$ & 38 & 8 & 21.05 & 1 & 2.63 & 0 & 0 \\
\hline $\mathrm{K}$ & 41 & 8 & 19.51 & 2 & 4.88 & 0 & 0 \\
\hline $\mathrm{L}$ & 37 & 5 & 13.51 & 2 & 5.41 & 2 & 5.41 \\
\hline $\mathrm{M}$ & 38 & 9 & 23.68 & 4 & 10.53 & 1 & 2.63 \\
\hline $\mathrm{N}$ & 37 & 9 & 24.32 & 5 & 13.51 & 1 & 2.70 \\
\hline $\mathrm{O}$ & 43 & 11 & 25.58 & 6 & 13.95 & 4 & 9.30 \\
\hline $\mathrm{P}$ & 40 & 11 & 27.5 & 5 & 12.5 & 0 & 0 \\
\hline Q & 39 & 10 & 25.64 & 6 & 15.38 & 4 & 10.26 \\
\hline $\mathrm{R}$ & 43 & 8 & 18.6 & 2 & 4.65 & 0 & 0 \\
\hline$S$ & 45 & 9 & 20.0 & 3 & 6.67 & 0 & 0 \\
\hline $\mathrm{T}$ & 39 & 8 & 20.51 & 1 & 2.56 & 0 & 0 \\
\hline $\mathrm{U}$ & 28 & 5 & 17.86 & 2 & 7.14 & 2 & 7.14 \\
\hline $\mathrm{V}$ & 7 & 2 & 28.57 & 1 & 14.29 & 0 & 0 \\
\hline $\mathrm{W}$ & 43 & 8 & 18.60 & 2 & 4.65 & 1 & 2.33 \\
\hline $\mathrm{X}$ & 35 & 7 & 20.0 & 1 & 2.86 & 0 & 0 \\
\hline $\mathrm{Y}$ & 39 & 6 & 15.38 & 2 & 5.13 & 0 & 0 \\
\hline$Z$ & 37 & 5 & 13.51 & 1 & 2.70 & 0 & 0 \\
\hline
\end{tabular}


From above table, it can be seen that the recognition accuracy by using PCA is much higher than that by using pixel template matching, and the recognition accuracy by using 2DPCA is higher than that by using PCA. But the similar characters can not be exactly distinguished even by using 2DPCA. Therefore, multi-level 2DPCA recognition is applied according Table 1. The recognition results are shown in Table 3 , it can be seen that the recognition accuracy to similar characters increase much, the accuracy can meet the recognition requirement.

Table 3 Results of recognition with multi-levels 2DPCA method

\begin{tabular}{|c|c|c|c|}
\hline \multirow{2}{*}{ Character } & \multirow{2}{*}{ Quantity } & \multicolumn{2}{|c|}{ Errors of multi level 2DPCA } \\
\cline { 3 - 4 } & & Quantity & Ratio (\%) \\
\hline 0 & 102 & 2 & 1.96 \\
\hline 1 & 85 & 0 & 0 \\
\hline 3 & 86 & 1 & 1.16 \\
\hline 6 & 80 & 2 & 2.50 \\
\hline 8 & 78 & 2 & 2.56 \\
\hline 9 & 95 & 2 & 2.11 \\
\hline B & 49 & 1 & 2.04 \\
\hline C & 38 & 0 & 0 \\
\hline D & 45 & 1 & 2.22 \\
\hline G & 39 & 1 & 2.56 \\
\hline L & 37 & 1 & 2.70 \\
\hline M & 38 & 0 & 0 \\
\hline N & 37 & 0 & 0 \\
\hline H & 38 & 0 & 0 \\
\hline O & 43 & 1 & 2.33 \\
\hline Q & 39 & 1 & 2.56 \\
\hline U & 28 & 0 & 0 \\
\hline
\end{tabular}

\section{Conclusion}

According to the characteristics of paper currency image, the extraction method of serial number region is researched. Sobel operator is first used to get the image edge, and then morphology close operation is applied to get serial number region exactly.

As the CIS image serial numbers have low resolution and they are easily polluted by noisy, multi-level 2DPCA algorithm is applied for serial number recognition. Experiment results show that the algorithm proposed in this paper not only costs little time but also has higher accuracy than pixel template matching, PCA and pure 2DPCA algorithm. Therefore, this algorithm is suitable for distinguishing similar characters.

\section{Acknowledgement}

In this paper, the research was sponsored by the Science and Technology Fund Project of Zhaoqing University(Project No. 201440).

\section{References}

[1] Bin Duo, Xuefeng Wang. The design and realization of paper currency recognition system. Journal Harbin University of Science \& Technology, Vol.13, No.4(2008), p.10-13. (in Chinese)

[2] Ying Zhang, Xuebo Chen, Qinghong Wu, etc. Identification system of RMB paper currency, Chinese Journal of Scientific Instrument, Vol.25, No.4(2004), p.669-690. (in Chinese) 
[3] Gaoyu Zhang. A real-time template generation algorithm to identify RMB [J].Information technology,2013.11:15-18(in Chinese)

[4] Tong Li, Daisheng Luo, Zhengyong Wang. RMB number recognition system based on neural networks optimized by genetic algorithm[J], Journal of Sichuan University(Natural Science Edition),2007,40(4):812-816 (in Chinese)

[5] Junmei Li, Yihua Hu, Xiaohong Tao. Recognition method based on principal component analysis and back propagation neural network[J]. Infrared and Laser Engineering, 2005, 34(6):719-723. (in Chinese)

[6] Qingjiang $\mathrm{Wu}$, Xiaoyan Zhou, Wenming Zheng. Face recognition based on 2D-DWT and 2D-PCA[J]. Computer Application, 2006.9， 26(9):2089-2091. (in Chinese)

[7] Fubing Cheng, Xiuhong Cheng, Shengliang Zhang, Jingyu Yang. A Human Face Recognition Method Based on Modular 2DPCA[J]. Journal of Image and Graphics,2006.4, 11(4): 580-585. (in Chinese) 